\title{
QUASISIMILARITY DOES NOT PRESERVE THE HYPERLATTICE
}

\author{
DOMINGO A. HERRERO
}

\begin{abstract}
Two quasisimilar nilpotent Hilbert space operators of order three can have nonisomorphic hyperinvariant subspace lattices.
\end{abstract}

1. Introduction. Let $\mathcal{L}(\mathcal{H C})$ be the algebra of all (bounded linear) operators acting on the complex separable Hilbert space $\mathcal{H}$. If $X \in \mathcal{L}(\mathcal{H C})$ and Ker $X$ $=\operatorname{Ker} X^{*}=\{0\}$, then $X$ is called a quasiaffinity. If $A, B \in \mathcal{L}(\mathcal{H C})$ and there exist $X, Y$ such that $A X=X B$ and $Y A=B Y$, then $A$ and $B$ are said to be quasisimilar. It is known that if $A$ and $B$ are quasisimilar operators, and $A$ has a nontrivial hyperinvariant (i.e., invariant under the commutant $\mathbb{Q}^{\prime}(A)$ of $A$ ) subspace, then so does $B$ (see [4] , [7] , [9]). Furthermore, if $A$ is normal then quasisimilarity induces an injection from Hyperlat $A$ into Hyperlat $B$ (see [8]; Hyperlattice is an abbreviation for lattice of hyperinvariant subspaces), so one could expect that quasisimilar operators always have isomorphic hyperlattices. An example will show that this is not necessarily true, even for very simple operators.

The author wishes to thank the referee for correcting several misprints and for helpful suggestions.

2. The hyperlattice of certain nilpotent operators.

Lemma 1. Let $Q$ be a nilpotent operator of order three $\left(Q^{3}=0\right)$. Then $\operatorname{Ker} Q^{2}\left(\left(\operatorname{Ran} Q^{2}\right)^{-}\right.$, resp.) is a maximal (minimal, resp.) hyperinvariant subspace of $Q$.

Proof. Let

$$
Q=\left(\begin{array}{ccc}
0 & Q_{12} & Q_{13} \\
0 & 0 & Q_{23} \\
0 & 0 & 0
\end{array}\right)
$$

be the matrix of $Q$ with respect to the orthogonal direct sum decomposition $\mathcal{K}=\mathcal{H}_{1} \oplus \mathcal{F}_{2} \oplus \mathcal{F}_{3}$, where $\mathcal{F}_{1}=\operatorname{Ker} Q, \mathcal{H}_{2}=\operatorname{Ker} Q^{2} \ominus \operatorname{Ker} Q$ and $\mathcal{H}_{3}$ $=\mathcal{H} \ominus \operatorname{Ker} Q^{2}$. Then [3] $Q_{12}$ and $Q_{23}$ are injective operators and therefore their adjoints have dense ranges.

Received by the editors November 24, 1976 and, in revised form, January 27, 1977.

AMS (MOS) subject classifications (1970). Primary 47A15.

Key words and phrases. Hyperinvariant subspace lattice, Jordan operator, nilpotent operator, quassimilarity.

- American Mathematical Society 1977 
A straightforward computation shows that the commutant of $Q$ consists of all those operators $A \in \mathcal{L}(\mathcal{H})$ of the form

$$
A=\left(\begin{array}{ccc}
A_{11} & A_{12} & A_{13} \\
0 & A_{22} & A_{23} \\
0 & 0 & A_{33}
\end{array}\right)
$$

such that $A_{11} Q_{12}=Q_{12} A_{22}, A_{22} Q_{23}=Q_{23} A_{33}$ and $A_{11} Q_{13}+A_{12} Q_{23}=Q_{12} A_{23}$ $+Q_{13} A_{33}\left(A_{13}\right.$ can be arbitrarily chosen).

Let $\mathfrak{N} \in$ Hyperlat $Q$ and assume that $\mathscr{N}$ is not contained in $\operatorname{Ker} Q^{2}$; then there exists a vector $(e, f, g)$ in $\mathscr{N}\left(e \in \mathscr{K}_{1}, f \in \mathscr{K}_{2}, g \in \mathscr{K}_{3}\right)$ with $g \neq 0$. Let $A$ be as in (2) with $A_{j k}=0$ for $(j, k) \neq(1,3)$; then the hyperinvariance of $\mathscr{N}$ implies that $A(e, f, g)=\left(A_{13} g, 0,0\right) \in \mathfrak{R}$. Since $A_{13}$ can be arbitrarily chosen, we conclude that $\mathscr{H}_{1} \subset \mathfrak{N}$. Hence $(0, f, g) \in \mathfrak{K}$.

Since $\operatorname{Ran} Q_{23}^{*}$ is dense, there exists an $f_{0} \in \mathcal{K}_{2}$ such that $\left\langle Q_{23}^{*} f_{0}, g\right\rangle=1$. Let $f_{2}$ be an element of $\mathcal{K}_{2}$ and define $B_{23}=f_{2} \otimes Q_{23}^{*} f_{0}, B_{12}=Q_{12} f_{2} \otimes f_{0}$ (where $x \otimes y$ denotes the operator defined by $x \otimes y(z)=\langle z, y\rangle x$ ) and $B_{j k}=0$ for all $(j, k) \neq(1,2)$ or $(2,3)$. It is easily seen that $B=\left(B_{j k}\right) \in$ $\mathbb{Q}^{\prime}(Q)$ and therefore $B(0, f, g)=\left(B_{12} f, B_{23} g, 0\right)=\left(B_{12} f, f_{2}, 0\right) \in \Re$, whence it readily follows that $\mathcal{K}_{1} \oplus \mathcal{K}_{2}=\operatorname{Ker} Q^{2} \subset \mathfrak{R}$. Hence $(0,0, g) \in$ T.

Now use the fact that $\operatorname{Ran}\left(Q_{12} Q_{23}\right)$ is dense in order to obtain an $e_{0} \in \mathcal{H}_{1}$ such that $\left\langle\left(Q_{12} Q_{23}\right)^{*} e_{0}, g\right\rangle=1$. Let $g_{3}$ be an element of $\mathscr{K}_{3}$ and define $C_{33}=g_{3} \otimes\left(Q_{12} Q_{23}\right)^{*} e_{0}, C_{22}=Q_{23} g_{3} \otimes Q_{12}^{*} e_{0}, C_{11}=Q_{12} Q_{23} g_{3} \otimes e_{0}, C_{12}=$ $Q_{13} g_{3} \otimes Q_{12}^{*} e_{0}, \quad C_{23}=Q_{23} g_{3} \otimes Q_{13}^{*} e_{0}$ and $C_{j k}=0$ for all $(j, k) \neq$ $(1,1),(1,2),(2,2),(2,3)$ or $(3,3)$. Then $C=\left(C_{j k}\right) \in \mathbb{Q}^{\prime}(Q)$ and therefore $C(0,0, g)=\left(0, C_{23} g, C_{33} g\right)=\left(0, C_{23} g, g_{3}\right) \in \mathfrak{R}$, whence we conclude that $\mathfrak{N}=\mathfrak{H}$.

The same arguments applied to $Q^{*}$ shows that $\operatorname{Ker} Q^{* 2}$ is a maximal hyperinvariant subspace of $Q^{*}$ and therefore $\left(\operatorname{Ker} Q^{* 2}\right)^{\perp}=\left(\operatorname{Ran} Q^{2}\right)^{-}$is a minimal hyperinvariant subspace of $Q$.

Corollary 2. If $\mathfrak{N} \in$ Hyperlat $Q, Q^{3}=0$ and $\mathscr{N} \neq\{0\}, \mathcal{H}$, then

$$
\left(\operatorname{Ran} Q^{2}\right)^{-} \subset \mathfrak{N} \subset \operatorname{Ker} Q^{2}
$$

Let $q_{k} \in \mathcal{L}\left(\mathbf{C}^{k}\right)$ be the nilpotent operator defined by $q_{k} e_{1}=0, q_{k} e_{j}=e_{j-1}$ for $j=2,3, \ldots, k$, with respect to the canonical ONB $\left\{e_{j}\right\}_{j=1}^{k}$ of $\mathbf{C}^{k}$ and let $q_{k}\left(\alpha_{k}\right)$ be the orthogonal direct sum of $\alpha_{k}$ copies of $q_{k}$ acting in the usual fashion on the orthogonal direct sum of $\alpha_{k}$ copies of $\mathbf{C}^{k}$. An operator $J \in \mathcal{L}(\mathcal{H})$ is a Jordan operator [2] if it can be written as $J=\bigoplus_{k=1}^{n} q_{k}\left(\alpha_{k}\right)$ with respect to a suitable decomposition

$$
\mathcal{H}=\bigoplus_{k=1}^{n}\left(\bigoplus_{j=1}^{\alpha_{k}} \mathbf{C}_{(j)}^{k}\right)
$$

of $\mathcal{K}$.

Lemma 3. Let $T \in \mathcal{C}(\mathcal{H})$, let $E \in \mathbb{Q}^{\prime}(T)$ be an idempotent operator and let 
$\Re \in$ Hyperlat $T$; then $E \Re=\Re \cap \operatorname{Ran} E \in$ Hyperlat $T_{E}$, where $T_{E}$ denotes the restriction of $T$ to $\operatorname{Ran} E$.

Let $\Re$ be a subspace and let $\left\{E_{\nu}\right\}_{\nu \in \Gamma}$ be a uniformly bounded chain of idempotents in $\mathbb{Q}^{\prime}(T)$ (if $\nu<\mu$ in $\Gamma$, then $\operatorname{Ran} E_{\nu} \subset \operatorname{Ran} E_{\mu}$ ) such that $E_{\nu} \rightarrow I$ (strongly). If $\mathfrak{K}_{\nu}=E_{\nu} \Re^{-}$, then $\Re \in$ Hyperlat $T$ if and only if $\mathfrak{\Re}_{\nu} \in$ Hyperlat $T_{E_{v}}$ for all $\nu \in \Gamma$.

Proof. Since $E=E^{2} \in \mathbb{Q}^{\prime}(T)$, for every $A \in \mathbb{Q}^{\prime}(T)$ the restriction $A_{E}$ of $E A E$ to $\operatorname{Ran} E$ belongs to $\mathbb{Q}^{\prime}\left(T_{E}\right)$.

Conversely, if $B_{0} \in \mathbb{Q}^{\prime}\left(T_{E}\right)$, then $B_{0}$ can be extended to an element $B$ of $Q^{\prime}(T)$ defined by $B x=B_{0} x$ if $x \in \operatorname{Ran} E$ and $B x=0$ if $x \in \operatorname{Ran}(I-E)$. It readily follows that $(E \mathscr{N})^{-} \in$ Hyperlat $T_{E}$. On the other hand, it is easily seen that $E \Re=\mathscr{R} \cap \operatorname{Ran} E$ and therefore $E \mathscr{N}$ is closed. This proves the first statement.

For the second one it is enough to observe that $E_{v} A E_{v} \rightarrow A$ for every $A \in \mathcal{L}(\mathcal{H})$. Hence $A \in \mathbb{Q}^{\prime}(T)$ if and only if $E_{\nu} A E_{\nu} \mid \operatorname{Ran} E_{\nu} \in \mathbb{Q}^{\prime}\left(T_{E_{\nu}}\right)$ for all $\nu$, whence the result follows.

PROPOSITION 4. If $J=\bigoplus_{k=1}^{n} q_{k}\left(\alpha_{k}\right)$ is a Jordan operator, then Hyperlat $J$ is the lattice generated by $\left\{\operatorname{Ker} J^{k}, \operatorname{Ran} J^{k}\right\}(k=0,1,2, \ldots, n)$ and it is orderisomorphic with Hyperlat $\bigoplus_{h=1}^{m} q_{k_{h}}$, where $\left\{k_{h}\right\}_{h=1}^{m}$ is the subset of $\{1,2, \ldots, n\}$ corresponding to those indices such that $\alpha_{k} \neq 0$.

Proof. Let $P_{N}$ be the orthogonal projection of $\mathcal{H}$ onto

$$
\mathcal{H}_{N}=\bigoplus_{k=1}^{n}\left(\bigoplus_{j=1}^{\min \left[\alpha_{k}, N\right]} \mathbf{C}_{(j)}^{k}\right)
$$

and let $J_{N}$ be the restriction of $J$ to $\mathcal{H}_{N}$.

Then Hyperlat $J_{N}$ is generated by $\left\{\operatorname{Ker} J_{N}^{k}, \operatorname{Ran} J_{N}^{k}: k=0,1,2, \ldots, n\right\}$ and it is order-isomorphic with Hyperlat $\bigoplus_{h=1}^{m} q_{k_{h}}$ (see [1], [5]). Now the result follows from the analysis of the hyperlattice of a finite dimensional operator carried out in the above references and Lemma 3.

3. The example. According to [2, Theorem 1] (or [10]), every nilpotent operator in $\mathcal{L}(\mathcal{H})$ is quasisimilar to a Jordan operator.

Let $\mathcal{K}=\mathrm{C} \oplus l_{1}^{2} \oplus l_{2}^{2} \oplus l_{3}^{2}$ and let $T \in \mathcal{L}(\mathcal{H})$ be the operator defined by the matrix

$$
T=\left(\begin{array}{cccc}
0 & 0 & 0 & T_{13 a} \\
0 & 0 & T_{12 b} & 0 \\
0 & 0 & 0 & T_{23} \\
0 & 0 & 0 & 0
\end{array}\right)
$$

where $T_{13 a}\left(\left\{c_{n}\right\}\right)=\sum_{n=1}^{\infty} c_{n} / n\left(T_{13 a}: l_{3}^{2} \rightarrow \mathrm{C}\right), T_{12 b}\left(\left\{c_{n}\right\}\right)=\left\{c_{n}\right\}\left(T_{12 b} ; l_{2}^{2} \rightarrow\right.$ $\left.l_{1}^{2}\right)$ and $T_{23}\left(\left\{c_{n}\right\}\right)=\left\{c_{n} / n^{2}\right\}\left(T_{23}: l_{3}^{2} \rightarrow l_{2}^{2}\right)$.

Proposition 5. Hyperlat $T$ is the chain of five elements $\{0\} \subset\left(\operatorname{Ran} T^{2}\right)^{-} \subset$ $\operatorname{Ker} T \subset(\operatorname{Ran} T)^{-}=\operatorname{Ker} T^{2} \subset \mathcal{H}$. 
COROllaRy 6. There exist two quasisimilar nilpotent operators $T$ and $J$ of order three such that Hyperlat $T$ and Hyperlat $J$ do not contain the same (finite) number of elements. In particular, these lattices are not order-isomorphic.

Proof. Choose $T$ given by (3). It is easily seen that $T^{3}=0$, so that ([2, Theorem 1]; see also [6], [10]) there exists a Jordan operator $J$ quasisimilar to $T$.

By Proposition 5, Hyperlat $T$ has five elements, but, by Proposition 4 and the results of [1], [5], Hyperlat $J$ can only have four, six or eight elements.

Proof of Proposition 5. $\left(\operatorname{Ran} T^{2}\right)^{-}=\left(\operatorname{Ran} T_{12 b} T_{23}\right)^{-}=l_{1}^{2}, \operatorname{Ker} T=\mathbf{C}$ $\oplus l_{1}^{2}, \operatorname{Ker} T^{2}=\mathrm{C} \oplus l_{1}^{2} \oplus l_{2}^{2}$ and $\operatorname{Ran} T=\left\{T_{13 a} g, T_{12 b} f, T_{23} g, 0: f \in l_{2}^{2}, g \in\right.$ $\left.l_{3}^{2}\right\}$. Clearly, $l_{1}^{2} \subset \operatorname{Ran} T$. Let $f_{1}=\left\{c_{1}, c_{2}, \ldots, c_{N}, 0,0, \ldots\right\} \in l_{2}^{2}$, let $\lambda \in \mathbf{C}$ and choose $g_{n}=\left\{c_{1}, 4 c_{2}, \ldots, N^{2} c_{N}, 0,0, \ldots, 0, d_{n}, 0, \ldots\right\} \in l_{3}^{2}$, where $d_{n}$ $=n\left(\lambda-\sum_{j=1}^{N} j c_{j}\right), n=1,2, \ldots ;$ then

$$
\begin{aligned}
& \| T\left(0,0,0, g_{n}\right)-\left(\lambda, 0, f_{1}, 0\right) \|=\left|d_{n} / n^{2}\right| \\
& \leqslant(1 / n)\left(|\lambda|+\sum_{j=1}^{N} j\left|c_{j}\right|\right) \rightarrow 0 \quad(n \rightarrow \infty) .
\end{aligned}
$$

Therefore $\mathbf{C} \oplus l_{2}^{2} \subset(\operatorname{Ran} T)^{-}$, i.e. $(\operatorname{Ran} T)^{-}=\mathbf{C} \oplus l_{1}^{2} \oplus l_{2}^{2}=\operatorname{Ker} T^{2}$.

Let $\Re \in$ Hyperlat $T, \mathscr{N} \neq\{0\}, \mathcal{H}$. By Corollary 2, $\left(\operatorname{Ran} T^{2}\right)^{-} \subset \Re \subset$ $\operatorname{Ker} T^{2}$. Assume that $(\lambda, 0, f, 0) \in \mathfrak{N}$ for some $f \in l_{2}^{2}, f \neq 0$, and let $\left\{f_{m}\right\}_{m=1}^{\infty}$ be the canonical ONB of $l_{2}^{2}$. Let $p$ be the first nonzero coordinate of $f$, i.e., $\left\langle f, f_{p}\right\rangle \neq 0$ and define

$$
A_{m}=\left(\begin{array}{cccc}
0 & 0 & A_{12 a}(m) & 0 \\
0 & A_{11 b}(m) & 0 & 0 \\
0 & 0 & A_{22}(m) & 0 \\
0 & 0 & 0 & A_{33}(m)
\end{array}\right)
$$

where $A_{11 b}(m)=A_{22}(m)=f_{m} \otimes f_{p}, A_{33}(m)=(m / p)^{2} f_{m} \otimes f_{p}$ and $A_{12 a}(m)\left(\left\{c_{n}\right\}\right)=m c_{p}, \quad m=1,2,3, \ldots$ It can be easily checked that $\left\{A_{m}\right\}_{m=1}^{\infty} \subset Q^{\prime}(T)$ and that $A_{m}(\lambda, 0, f, 0)=\left(A_{12 a}(m) f, 0, A_{22}(m) f, 0\right)=$ $\left\langle f, f_{p}\right\rangle\left(m, 0, f_{m}, 0\right) \in \Re$.

Hence, $\left(1 / m\left\langle f, f_{p}\right\rangle\right) A_{m}(\lambda, 0, f, 0) \rightarrow(1,0,0,0)(m \rightarrow \infty)$ and therefore $\mathbf{C}$ $\subset \Re$. A fortiori, $\left(0,0, f_{m}, 0\right)=\left(m, 0, f_{m}, 0\right)-(m, 0,0,0) \in \Re$ and therefore $\Re=\mathrm{C} \oplus l_{1}^{2} \oplus l_{2}^{2}=\operatorname{Ker} T^{2}$.

On the other hand, if $(\lambda, 0, f, 0) \in \Re$ implies $f=0$, then it is easy to see that either $\mathscr{K}=l_{1}^{2}=(\operatorname{Ran} T)^{-}($and $\lambda \equiv 0)$ or $\mathscr{N}=\mathrm{C} \oplus l_{1}^{2}=\operatorname{Ker} T$.

\section{REFERENCES}

1. T. Andô and T. Sekiguchi, Hyperinvariant subspaces of a nilpotent operator (preprint).

2. C. Apostol, R. G. Douglas and C. Foias, Quasisimilar models for nilpotent operators (to appear). 
3. J. Barria and D. A. Herrero, Closure of similarity orbits of nilpotent operators. II (preprint).

4. P. A. Fillmore, Notes in operator theory, Mathematical Studies No. 30, Van Nostrand, Princeton, N.J., 1970.

5. P. A. Fillmore, D. A. Herrero and W. E. Longstaff, The hyperinvariant subspace lattice of a linear transformation, Linear Algebra and Appl. (to appear).

6. C. Foiaș and C. Pearcy, A model for quasinilpotent operators, Michigan Math. J. 21 (1974), 399-404.

7. T. B. Hoover, Quasisimilarity of operators, Illinois J. Math. 16 (1972), 678-686. MR 47 \#866.

8. __ Hyperinoariant subspaces for n-normal operators, Acta Sci. Math. (Szeged) 32 (1971), $109-119$.

9. B. Sz.-Nagy and C. Foias, Analyse harmonique des opérateurs de l'espace de Hilbert, Masson et Cie., Paris, Akademiai Kiadó, Budapest, 1967. MR 37 \#778.

10. L. Williams, Similarity invariants for a class of nilpotent operators, Acta Sci. Math. (Szeged) (to appear).

Departamento de Mathematicas, Instituto Venezolano de Investigaciones Científicas (I.V.I.C.), Apartdo Postal 1827, Caracas, Venezuela 